\title{
Fusobacterium nucleatum bacteremia with liver abscess following administration of anti-COVID-19 vector-based vaccine
}

\author{
Jiun-Ting $\mathrm{Wu}^{1}$, Yung-Fa $\mathrm{Lai}^{2}$, and Chien-Tung $\mathrm{Chiu}^{2}$ \\ ${ }^{1}$ E-DA cancer Hospital \\ ${ }^{2}$ E-DA Hospital
}

January 26, 2022

\begin{abstract}
Immunization of the population through vaccination against COVID-19 is recognized as a public health priority. The most difficult scientific challenge for vaccines is the clinical safety and efficacy. We report a patient presenting with Fusobacterium nucleatum bacteremia and liver abscess formation following administration of anti-COVID-19 viral-vectored vaccine.
\end{abstract}

\section{Introduction}

Fusobacterium species are anaerobic, Gram-negative, rod-shaped, adherent, non-motile and non-sporeforming bacteria.Fusobacterium nucleatum is an oral bacterium, commensal to the human oral cavity, that plays a role in periodontal disease and colorectal neoplasm ${ }^{1,2} . F$. nucleatum is a rare cause of bacteremia; the annual reported incidence is $0.22-0.76$ cases/100,000 population ${ }^{3,4}$. Risk factors for $F$. nucleatum bacteremia include malignancy, older age, alcohol abuse, immunosuppression, and dialysis; infection is often hospital-acquired ${ }^{3}$.

Immunization of the population through vaccination is recognized as a public health priority against COVID19. The clinical efficacy and safety for vaccines against the novel coronavirus is the most difficult scientific challenge at present. We report the case of a 67-year-old man, an immunocompetent patient with $F$. nucleatum bacteremia and liver abscess formation following administration of the first dose of anti-COVID19 viral-vectored vaccine during the COVID-19 pandemic.

\section{Case history}

A 67-year-old physician with a past history of hypertension, allergic rhinitis and hepatic hemagioma for 10 years received the first dose of ChAdOx1nCov-19 vaccine on 2021/3/22. Three days later, he presented with fever, up to $39^{\circ} \mathrm{C}$, combined with general soreness. He took an acetaminophen tablet and his fever subsided completely. However, fever up 39.3 with chills occurred on $3 / 31$, and he was admitted for detailed evaluation. Blood culture was done. Neither leukocytosis nor a higher C-reactive protein (CRP) level was noted, and the Covid-19 PCR was negative. The next day, his fever subsided and he was discharged.

However, he developed a fever, up to 38.5, 2 days after discharge. Owing to persistent fever for 3 days, he subsequently visited the infectious disease outpatient department and was admitted for further evaluation. On admission, he had a temperature of 37.6 , blood pressure of $127 / 71 \mathrm{mmHg}$, and heart rate of 80 beats per minute. On physical examination, he did not have a toxic appearance, but his tonsils had erythematous enlargement. No neck lymphadenopathy was found. His chest examination revealed clear breathing sounds, and a regular heart rhythm without murmur. His abdominal examination revealed a soft abdomen without rigidity or rebound, and no tenderness to palpation. There was no lower extremity edema. His skin was intact without rashes. His white blood cell count was $20.14 \times 10^{3} / \mu \mathrm{L}$ (reference range $3.8-10.8 \times 10^{3} / \mu \mathrm{L}$ ) 
with $86.8 \%$ neutrophils, hemoglobin was $12.9 \mathrm{~g} / \mathrm{dL}$ (reference range $12-15.5 \mathrm{~g} / \mathrm{dL}$ ), and platelet count was $246 \times 10^{3} / \mu \mathrm{L}$ (reference range $150-450 \times 10^{3} / \mu \mathrm{L}$ ). His liver chemistries revealed aspartate aminotransferase of $50 \mathrm{U} / \mathrm{L}$, alanine aminotransferase of $60 \mathrm{U} / \mathrm{L}$, and total bilirubin of $0.64 \mathrm{mg} / \mathrm{dL}$. Meanwhile, a CRP level of $148.56 \mathrm{mg} / \mathrm{L}$ (reference range $<5 \mathrm{mg} / \mathrm{L}$ ) and procalcitonin $\mathrm{C}$ of $3.1 \mathrm{ng} / \mathrm{ml}$ (reference range $<0.5 \mathrm{ng} / \mathrm{mL}$ ) were found, which revealed his inflammatory markers were significantly more elevated than during the previous admission. His COVID-19 nasopharyngeal swab test was still negative. Both abdominal echography and computed tomography of the abdomen showed $4.5-\mathrm{cm}$ abscesses at left lateral segment of the liver (Figure 1). Blood cultures were obtained and he was given empiric antibiotic treatment. The liver abscess was drained with a pigtail catheter on the next day. The pus smear revealed Gram-negative bacilli. Later, his blood cultures, which had been collected during the previous admission, yieldedFusobacterium nucleatum (F. nucleatum ). He then underwent a colonoscopy examination, which revealed diverticulosis at the cecum and ascending colon. A dentist was consulted and asymptomatic periodontitis was found.

The patient then received antibiotics with metronidazole and underwent liver abscess drainage. His fever subsided completely and he was discharged under a stable status. After 4 weeks of metronidazole treatment, the patient was able to return to his normal daily life.

\section{Discussion:}

Fusobacterium species are Gram-negative anaerobic bacilli which are normally a constituent of the oropharynx, gastrointestinal tract, and female genital flora. Overall, Fusobacterium species are a rare cause of bacteremia, accounting for $<0.001 \%$ of all bacteremia and $<1 \%$ of anaerobic bacteremia cases among adults $^{5}$. Fusobacterium bacteremia generally affects males more than females ${ }^{6,7}$, with the primary infection source typically being the respiratory tract, abdomen, or pelvis ${ }^{6,8}$. With respect to the hepatic abscesses associated with Fusobacterium species, oropharyngeal disease or intestinal sources of infections including diverticulitis have been postulated as the potential initial portal of entry ${ }^{7}$. Our patient had periodontal disease, but no evidence of oropharyngeal infections was noted.

Was this bacteremia event a coincidence or a complication related to vaccination? F. nucleatum is an adhesive bacterium. It can co-aggregate with various microbial species in the oral cavity, and plays a key role in dental plaque formation ${ }^{7,9}$. In healthy individuals, there is an established homeostasis between immunity and oral cavity microorganisms that do not cause diseases. Once disseminated outside the oral cavity and under dysbiosis, F. nucleatum induces exacerbated inflammation, thus turning into a pathogen ${ }^{10,11}$. Regulatory $\mathrm{T}$ cells (Tregs) have been known to act on the balance of immunopathogenesis of periodontal lesions. When Tregs function was inhibited, it increased alveolar bone loss and inflammatory cell migration, and as such, the tissue damage associated with periodontitis was noted ${ }^{12}$. Furthermore, $F$. nucleatum stimulates Toll-like receptor 4 (TLR4)-mediated inflammatory responses in the placentas of pregnant mice, causing fetal demise. Suppression of inflammation protects the fetuses, even in the presence of bacterial colonization ${ }^{13}$. However, the exact molecular immunological pathway against $F$. nucleatum is not fully understood. Therefore, we supposed that our patient experienced both wanted and unwanted immunogenicity and the disruption of symbiont status after vaccination, which led to $F$. nucleatum bacteremia.

Fever developed off and on in our patient about 1 week after vaccination. All laboratory data initially showed no abnormality, and he was treated as having an adverse effect post-vaccination. Then, on day 3 of his second admission, the blood culture that was obtained during his first hospitalization grew $F$. nucleatum - The Oxford AstraZeneca chimpanzee adenovirus-vectored vaccine, ChAdOx1 nCoV-19, has appeared to be effective and safe for SARS-CoV-2 immunization ${ }^{14}$. The incidence of objectively measured fever was low in the 18-55 years standard-dose group (12 [24\%] of 49), and no fevers were recorded in either the 56-69 years or older standard-dose groups within 7 days after the prime vaccination with ChAdOx1 nCoV- $19^{14}$. Therefore, the diagnosis of systemic adverse reactions post-vaccination should be determined carefully by a clinical physician, especially fever occurrence during an unexpected period among the elderly patient group.

During the COVID-19 pandemic, the normal processes of clinical inference and diagnosis were disrupted and delayed treatment of bacteremia has been noted ${ }^{15}$. Although $F$. nucleatum bacteremia associated with 
coronavirus disease was reported recently during the COVID-19 pandemic ${ }^{16}$, to the best of our knowledge, this is the first case report of $F$. nucleatum bacteremia with hepatic abscess in an immunocompetent patient post-anti-COVID-19 vaccine administration. The exact relationship between host immunological reaction and the pathogenesis of bacteremia is difficult to identify at present, so further research is needed to elucidate this interaction.

\section{Authorship}

\section{AUTHOR CONTRIBUTIONS}

Jiun-Ting Wu:contributed to the literature search, and wrote the manuscript

Yung-Fa Lai:provided the concept and design of this report

Chien-Tung Chiu: qualified the patient's image

All authors approved the final version of the manuscript.

Ethical approval

The patient gave permission and informed consent for the publication of this case report.

\section{Funding source:}

This research did not receive any specific grant from funding agencies in the public, commercial, or not-forprofit sectors.

\section{ACKNOWLEDGEMENT}

NA. Published with written consent of the patient.

\section{Conflict of Interest Statement}

The authors have no conflicts of interest to disclose.

\section{References:}

1. Kapatral V, Anderson I, Ivanova N, Reznik G, Los T, Lykidis A, et al. Genome Sequence and Analysis of the Oral Bacterium $<\mathrm{i}>$ Fusobacterium nucleatum $</ \mathrm{i}>$ Strain ATCC 25586. Journal of Bacteriology. 2002;184(7):2005-18.

2. Castellarin M, Warren RL, Freeman JD, Dreolini L, Krzywinski M, Strauss J, et al. Fusobacterium nucleatum infection is prevalent in human colorectal carcinoma. Genome research. 2012;22(2):299-306.

3. Afra K, Laupland K, Leal J, Lloyd T, Gregson D. Incidence, risk factors, and outcomes of Fusobacterium species bacteremia. BMC Infect Dis. 2013;13:264.

4. Pett E, Saeed K, Dryden M. Fusobacterium species infections: clinical spectrum and outcomes at a district general hospital. Infection. 2014;42(2):363-70.

5. Dorsher CW, Rosenblatt JE, Wilson WR, Ilstrup DM. Anaerobic Bacteremia: Decreasing Rate Over a 15-Year Period. Reviews of Infectious Diseases. 1991;13(4):633-6.

6. Anne-Marie B, Fran, xe, ois L, Patrick D, xe, et al. Fusobacterium Bacteremia: Clinical Experience with 40 Cases. Clinical Infectious Diseases. 1997;25:S181-S3.

7. Huggan PJ, Murdoch DR. Fusobacterial infections: Clinical spectrum and incidence of invasive disease. Journal of Infection. 2008;57(4):283-9.

8. Goldberg EA, Venkat-Ramani T, Hewit M, Bonilla HF. Epidemiology and clinical outcomes of patients with Fusobacterium bacteraemia. Epidemiology and Infection. 2013;141(2):325-9. 
9. Rickard AH, Gilbert P, High NJ, Kolenbrander PE, Handley PS. Bacterial coaggregation: an integral process in the development of multi-species biofilms. Trends Microbiol. 2003;11(2):94-100.

10. Brennan CA, Garrett WS. Fusobacterium nucleatum - symbiont, opportunist and oncobacterium. Nat Rev Microbiol. 2019;17(3):156-66.

11. de Andrade KQ, Almeida-da-Silva CLC, Coutinho-Silva R. Immunological Pathways Triggered by Porphyromonas gingivalis and Fusobacterium nucleatum: Therapeutic Possibilities? Mediators Inflamm. 2019;2019:7241312.

12. Garlet GP, Cardoso CR, Mariano FS, Claudino M, De Assis GF, Campanelli AP, et al. Regulatory T cells attenuate experimental periodontitis progression in mice. Journal of clinical periodontology. 2010;37(7):591600 .

13. Liu H, Redline RW, Han YW. <em $>$ Fusobacterium nucleatum $</$ em $>$ Induces Fetal Death in Mice via Stimulation of TLR4-Mediated Placental Inflammatory Response. The Journal of Immunology. 2007;179(4):2501-8.

14. Ramasamy MN, Minassian AM, Ewer KJ, Flaxman AL, Folegatti PM, Owens DR, et al. Safety and immunogenicity of ChAdOx $1 \mathrm{nCoV}-19$ vaccine administered in a prime-boost regimen in young and old adults (COV002): a single-blind, randomised, controlled, phase 2/3 trial. The Lancet. 2020;396(10267):1979-93.

15. Miyagami T, Uehara Y, Harada T, Watari T, Shimizu T, Nakamura A, et al. Delayed treatment of bacteremia during the COVID-19 pandemic. Diagnosis. 2021(000010151520200114).

16. Wolff L, Martiny D, Deyi VYM, Maillart E, Clevenbergh P, Dauby N. COVID-19-Associated Fusobacterium nucleatum Bacteremia, Belgium. Emerg Infect Dis. 2021;27(3):975-7.

\section{Hosted file}

Figure.docx available at https://authorea.com/users/457346/articles/554163-fusobacteriumnucleatum-bacteremia-with-liver-abscess-following-administration-of-anti-covid-19-

vector-based-vaccine 